\title{
Clostridium difficile auf dem Vormarsch
}

\author{
Eine große Studie zeigt, dass Infektionen mit dem Erreger Clostridium difficile häufiger werden \\ - gerade solche mit kompliziertem Verlauf. Für niedergelassene Ärzte ist das ein Warnsignal.
}

\begin{abstract}
_ Die aktuell beobachtete Zunahme von Infektionen mit Clostridium (C.) difficile liegt nicht nur an der steigenden Anzahl älterer und multimorbider Patienten, sondern auch an der nach wie vor zunehmenden Verschreibung von Antibiotika und dem vermehrten Auftreten von C.-difficile-Stämmen mit höherer Virulenz. In der Folge gibt es auch immer mehr komplizierende Verläufe und Rezidive, insbesondere multiple - was bisher allerdings lediglich aus Fallbeschreibungen und kleineren epidemiologischen Studien bekannt war.

Eine große epidemiologische Studie aus den USA beschreibt nun, dass in den
\end{abstract}

Jahren 2000-2010 eine Verdoppelung der stationären Aufenthalte aufgrund einer Infektion mit C. difficile zu beobachten war. Im gleichen Zeitraum verdreifachte sich die Zahl der stationär aufgenommen Patienten mit einem toxischen Megakolon - ein eindrucksvoller Hinweis darauf, dass die komplizierten Verläufe überproportional zunehmen. Die Mortalität des C.-difficile-assoziierten toxischen Megakolon stieg von $13,6 \%$ auf $24,5 \%$.

- Kuy S, Jenkins P, Ramon A et al. Increasing incidence of and increased mortality associated with clostridium difficile-associated megacolon. JAMA Surg. 2015; online 7. Oktober, doi: 10.1001/jamasurg.2015.2677

\section{KOMMENTAR}

In Kenntnis dieser Daten sollten Infektionen mit C. difficile vermieden bzw. frühzeitig erkannt werden - durch einen rationalen Einsatz von Antibiotika, geeigneten Hygienemaßnahmen und gesteigerte Aufmerksamkeit. Insbesondere bei Risikopatienten sollten durch eine zeitnahe und zielgerichtete Diagnostik sowie Therapie schwere und rezidivierende Verläufe abgewendet werden. Durch einen einfachen Stuhltest kann die Diagnose rasch gesichert werden. In dringenden Fällen erscheint auch ein Therapiebeginn vor Eintreffen des Laborergebnisses als ein probates Vorgehen.

Prof. Dr. med. Martin Storr

\section{Ein Ast beendet jäh den Ausflug mit dem Mountain Bike}

Ein 40-jähriger Mann stürzte mit dem Mountainbike im Gelände unglücklich und spießte sich dabei einen $2 \mathrm{~cm}$ dicken Ast in den Hals. Der Mann versuchte nicht, den Ast zu entfernen und war in der Lage, aus eigenen Kräften eine
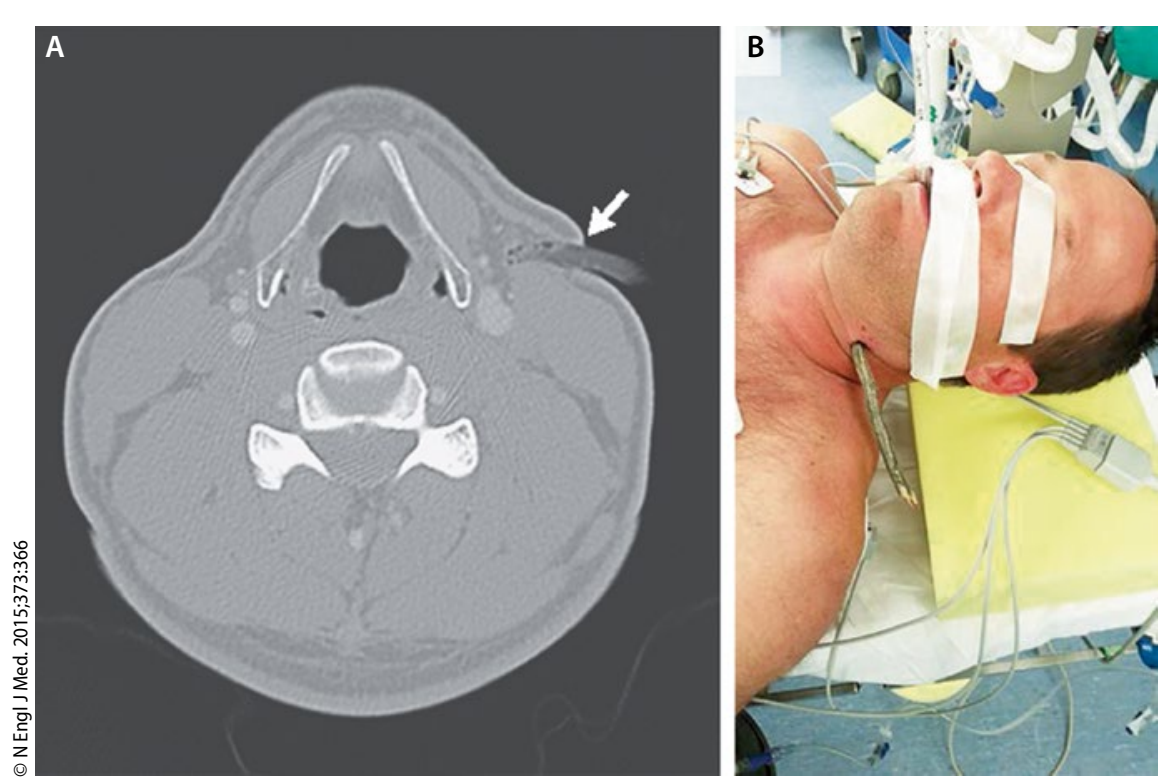

CT: Fremdkörper in der linken Halsseite im (A), Situs: herausragender Ast (B).

Nothilfe aufzusuchen. Im Computertomogramm stellte sich ein Fremdkörper in den Weichteilen der linken Halsseite in Höhe des Schildknorpels dar, der das Platysma durchbohrt hatte, $1,6 \mathrm{~cm}$ tief eingedrungen war und 